\title{
APRENDIENDO LA HISTORIA DE LA TIERRA Y DE LA VIDA A TRAVÉS DE LA MÚSICA
}

Learning the History of Earth and Life through music

Aprendendo a História da Terra e da Vida através da música

\section{María Iluminada Muñoz Lucas (1)}

Fernando José Pereira García (2)

(1) Departamento Científico-Tecnológico. Centro de Educación de Adultos Celtiberia, Soria, España. Teléfono: +34 691347534. Correo electrónico: minmaculada.mun@educa.jcyl.es

(2) Universidad de León, España. Teléfono: +34 987291535. Correo electrónico:

\section{fjperg@unileon.es}

\begin{abstract}
Resumen
Se propone una actividad que tiene como objetivo emplear las emociones transmitidas por la música para potenciar recuerdos sólidos y duraderos en los estudiantes. Trabajando con un grupo de alumnos de la materia de Biología y Geología de $4^{\circ}$ curso de ESO, se ha elaborado una lista de reproducción de fragmentos musicales evocadores de acontecimientos relevantes en la Historia de nuestro planeta y de la vida que éste alberga. La evaluación de esta buena práctica se ha llevado a cabo empleando un procedimiento de evaluación entre iguales, para el cual se ha diseñado una escala de valoración, y un reparto dialogado de puntuaciones consensuado a partir de cuestionarios de seguimiento intragrupal.

Palabras clave: Emociones; Biología y Geología; música; evaluación entre iguales; experiencia educativa
\end{abstract}

\section{Abstract}

An experience that aims to use the emotions transmitted by music to enhance solid and long lasting memories for students is proposed in this paper. Working with a group of students of Biology and Geology in 4th year of ESO, a list of evocative, musical fragments related to relevant events in the History of our planet and life has been elaborated. The evaluation process of this good practice has been carried out using a 
peer review process, for which it has been designed a rating scale, and using a consensual, dialogued distribution of scores employing intragroup monitoring questionnaires.

Keywords: Emotions; Biology and Geology; music; peer review process; educative experience

\section{Resumo}

Uma atividade que visa usar as emoções transmitidas pela música para melhorar memórias sólidas e duradouras para os alunos é proposta. Trabalhando com um grupo de estudantes na área de Biologia e Geologia $4^{\circ}$ ano de ESO, ele compilou uma lista de reprodução de fragmentos musicais evocativos de eventos importantes na história do nosso planeta e da vida que ele contém. A avaliação desta boa prática foi realizada utilizando um processo de revisão por pares, para o qual projetou uma escala de avaliação, e uma distribuição dialogada das pontuações com questionários de monitoramento intragrupo.

Palavras-chave: Emoções; Biologia e Geologia; música; processo de revisão por pares; experiência educacional

\section{Introducción}

Poner en práctica metodologías educativas y evaluaciones formativas innovadoras supone un cambio al estilo tradicional. A través de estas buenas prácticas sepretende generar un aprendizaje significativo en el alumnado (Ausubel, Novak, \& Hanesian, 1989). Para ello, es fundamental desarrollar actividades no sólo novedosas, sino también capaces de alcanzarlos objetivos planteados en las Programaciones Didácticas (Zabalza, 2012). Estos objetivos se pueden conseguir trabajando las competencias clave y centrando el aprendizaje en las Inteligencias Múltiples de nuestros estudiantes (Gardner, 1983).

La actividad que se propone se titula “Escuchando el transcurrir del tiempo" y tiene como objetivo conseguir que los alumnos desarrollen recuerdos sólidos y duraderos a partir de emociones intensas (Rodríguez Palmero, 2011), como las que son capaces de transmitir las artes, en este caso la música. Partiendo de esta premisa se ha 
trabajado anteriormente, con resultados muy satisfactorios, como los obtenidos por el profesor Richard Spencer (Velasco, 2015).

\section{Contextualización}

La actividad ha sido realizada por alumnos de la materia de Biología y Geología de $4^{\circ}$ de ESO Se trata de un grupo de veinticinco estudiantes con edades comprendidas entre quince y diecisiete años. El 12 \% son inmigrantes y acaban de llegar a España.

Esta actividad se engloba, actualmente, en los Bloques 1 y 2, La evolución de la vida y La dinámica de la Tierra, respectivamente, recogidos en el currículo de Biología y Geología de $4^{\circ}$ de ESO, según la Orden EDU/362/2015, de 4 de mayo, por la que se establece el currículo y se regula la implantación, evaluación y desarrollo de la Educación Secundaria Obligatoria en la Comunidad de Castilla y León.

\section{Diseño y desarrollo}

Esta experiencia ha sido diseñada teniendo en cuenta la amplia diversidad de estudiantes existente en el grupo (motivaciones, capacidades, trayectoria académica, etc.). Con el fin de favorecer el recuerdo a través de las emociones, la idea general de la actividad es invitar al alumnado a elegir fragmentos musicales que les evoquen eventos relevantes de la Historia de la Tierra y de la Vida. La duración prevista comprende las ocho sesiones correspondientes a la unidad que trata la temática citada anteriormente.

En la primera sesión, se presenta la actividad entregando a los discentes una relación de acontecimientos relevantes en la Historia de la Tierra y de la Vida, como pueden ser el Big Bang, la aparición de la vida en la Tierra, la fotosíntesis o el surgimiento de los primeros homínidos, los cuales van a ir conociendo a lo largo de la unidad. El listado está compuesto por quince eventos diferentes. Los alumnos deberán elegir los sucesos que deseen y asignarles un fragmento musical que asocien al mismo en función de lo aprendido, a fin de manifestar las emociones que les sugiere un hecho histórico a través del arte, en este caso la conciencia musical. La elección de las canciones será totalmente libre por parte de los estudiantes.

Esta propuesta se realiza en grupos de cinco alumnos, que trabajan en casa a lo largo de la unidad eligiendo, bajo su criterio, los mejores temas musicales. En dos sesiones intermedias, se reservan los diez últimos minutos para que el docente compruebe los avances de sus estudiantes, guiándolos si fuera necesario. 
Al concluir la actividad, se reproducen los temas de cada grupo en el aula y los alumnos justifican su elección, generando un debate acerca de las razones que les llevaron a decantarse por una u otra opción. Entre toda la clase deciden qué canciones definen mejor cada suceso y se crea, con ellas, una lista de reproducción en la plataforma Spotify, asociando cada tema a la relación de acontecimientos.

\section{Evaluación}

Se desarrolla mediante un procedimiento de evaluación entre iguales, ya que los estudiantes, siguiendo los procesos adecuados, aprenden mejor si los evalúa un semejante que si lo hace un docente (Antón-Nuño \& Moraza-Herrán, 2010; GómezRodríguez, Ibarra-Sáiz, \& García-Jiménez, 2013). Para que esta evaluación tenga éxito, los alumnos consensuan previamente con el profesor una escala de valoración (Tabla 1), básica para evaluar el trabajo del resto de grupos. Se ha optado por este instrumento debido a su capacidad para facilitar que los estudiantes emitan un juicio personal sobre el trabajo de sus compañeros (Fernández-March, 2008).

Tabla 1.

Escala de valoración para evaluar los grupos de trabajo.

\begin{tabular}{|c|c|}
\hline Número de acontecimientos & $\begin{array}{c}\text { Valoración } \\
\text { (puntos) }\end{array}$ \\
\hline 10 & 10 \\
\hline $8-9$ & 7 \\
\hline $6-7$ & 3 \\
\hline Menos de 6 & 1 \\
\hline Elección de los temas musicales & $\begin{array}{c}\text { Valoración } \\
\text { (puntos) }\end{array}$ \\
\hline Los temas propuestos están muy relacionados con los eventos correspondientes & 15 \\
\hline La mayoría de los temasse relacionan con los eventos correspondientes & 12 \\
\hline Pocos o ningún tema se relacionan con los eventos correspondientes & 5 \\
\hline Exposición & $\begin{array}{c}\text { Valoración } \\
\text { (puntos) }\end{array}$ \\
\hline Todos los miembros del grupo presentan sus canciones & 10 \\
\hline No todos los miembros del grupo presentansus canciones & 3 \\
\hline Sólo un miembro del grupo presenta sus canciones & 1 \\
\hline Debate & $\begin{array}{c}\text { Valoración } \\
\text { (puntos) }\end{array}$ \\
\hline $\begin{array}{l}\text { Todos los miembros del grupo participan en el debate. Respetan las intervenciones de } \\
\text { los otros grupos }\end{array}$ & 15 \\
\hline $\begin{array}{l}\text { Algunos miembros del grupo participan en el debate. Respetan las intervenciones de } \\
\text { los otros grupos }\end{array}$ & 10 \\
\hline $\begin{array}{l}\text { Sólo participa un miembro en el debate. Respetan las intervenciones de los otros } \\
\text { grupos }\end{array}$ & 7 \\
\hline $\begin{array}{l}\text { Todos o algunos miembros del grupo participan en el debate. No respetan al resto de } \\
\text { grupos }\end{array}$ & 2 \\
\hline Sólo participa un miembro en el debate. No respetan al resto de grupos & 1 \\
\hline TOTAL & 50 \\
\hline
\end{tabular}


De esta manera, los puntos obtenidos por cada grupo (entre 8 y 50) se multiplican, a continuación, por el número de miembros del equipo (lográndose de 40 a 250 puntos). La puntuación definitiva se reparte entre los integrantes del grupo en función de su trabajo individual, esfuerzo personal, implicación, etc. Para llevar a cabo este reparto dialogado de puntuaciones de forma correcta, se proporciona a cada estudiante un cuestionario de seguimiento intragrupal

\section{Conclusiones}

Desde la perspectiva del profesor, el desarrollo de esta actividad ha creado un clima distendido en el aula, incrementando el interés de los estudiantes por participar en ella. Además, los alumnos se han sentido cómodos a lo largo de la experiencia, con lo cual se ha favorecido la adquisición de recuerdos que, probablemente, perdurarán en el tiempo.

Se han trabajado las Inteligencias Múltiples musical, lingüística, intrapersonal e interpersonal, así como las siguientes competencias clave: competencias básicas en ciencia y tecnología, de conciencia y expresiones culturales, comunicación lingüística y competencia para aprender a aprender.

Una gran parte de los estudiantes ha manifestado que, en lo que respecta al trabajo de búsqueda de canciones en casa, sus familias se han involucrado de manera entusiasta y han compartido con ellos sus preferencias musicales. Este apoyo familiar puede repercutir directamente en el éxito escolar del alumno.

El interés suscitado por esta actividad, y en aras de mejorarla, lleva a plantear cambios futuros a través de la colaboración interdisciplinar del Departamento de Ciencias Naturales con el de Música y del ajuste del número de sesiones de evaluación, que en la práctica han resultado excesivas. Asimismo, se pretende transferir esta experiencia a la Educación Secundaria Para Adultos (ESPA) durante el curso lectivo 2016-2017.

\section{Referencias}

Antón-Nuño, A., \& Moraza-Herrán, J. I. (2010). Experiencias de coevaluación entre iguales: valoraciones de los alumnos y del profesor. INFAD Revista de Psicología, 2(12), 687-693.

Ausubel, D., Novak, J. D., \& Hanesian, H. (1989). Psicología cognitiva. México: Trilhas. 
Fernández-March, A. (2008). La evaluación de los aprendizajes en la universidad: nuevos enfoques. Instituto de Ciencias de la Educación, Universidad de Valencia. Retrieved from https://goo.gl/DHmMN3

Gardner, H. (1983). Frames of mind. The Theory of the Multiple intelligences. Nueva York: Basics.

Gómez-Rodríguez, G., Ibarra-Sáiz, M. S., \& García-Jiménez, E. (2013). Autoevaluación, evaluación entre iguales y coevaluación: conceptualización y práctica en las universidades españolas. Revista de Investigación en Educación, 11(2), 198-210.

Rodríguez Palmero, M. L. (2011). La teoría del aprendizaje significativo: una revisión aplicable a la escuela actual. IN. Investigació I Innovació Educativa I Socioeducativa, 3(1), 29-50.

Velasco, S. (2015). Enseñar biología con música y baile, por Richard Spencer. Retrieved from https://goo.gl/fSdxh7

Zabalza, M. A. (2012). El estudio de las "Buenas Prácticas” docentes en la enseñanza universitaria. REDU. Revista de Docencia Universitaria, 10(1), 17-42. 